\title{
Design of Bandpass Elliptic Filters Employing Inductive Windows and Dielectric Objects
}

\author{
Francisco Javier Pérez Soler, Mónica Martínez Mendoza \\ Fernando Daniel Quesada Pereira, David Cañete Rebenaque \\ Alejandro Alvarez Melcon, and Richard J. Cameron.
}

\begin{abstract}
In this paper, we propose an alternative configuration for the design of bandpass inductive filters using transversal topologies. The structure is based on the use of a dielectric post asymmetrically placed on a cavity resonator. The input and output windows will couple energy to the $T E_{102}$ - and $T E_{103}$-mode resonators at the same time, therefore obtaining the transversal topology. The proposed configuration is very compact, and allows to implement transmission zeros for maximum selectivity in an easy way. Results are validated with Ansoft's finite-element High Frequency Structure Simulator tool, and with an integral-equation technique to demonstrate the validity of the proposed structure.
\end{abstract}

Index Terms-Dielectric posts, doublet topology, inductive filters, transmission zeros, transversal filters, waveguide filters.

\section{INTRODUCTION}

$\mathbf{F}$ ILTER DESIGN is one of the most interesting fields in microwave engineering. A wide number of different topologies allows to obtain specific responses for a wide range of applications. Inductive filters constitute a strategy of special interest due to their simplicity and easy manufacturing processes associated with these configurations [1]. In this line, several studies have been developed in the past to improve the electrical characteristics of inductive waveguide filters. For instance, microwave filters with inductive windows were explored in [2] for space applications. In addition, inductive filters with metallic and dielectric posts have been studied in detail in [3]-[5]. Elliptic responses were also investigated, for the first time, combined with inductive topologies in [6]. The work presented in [6] is, in fact, very interesting since it constitutes the first attempt to implement transversal filters with inductive waveguide topologies.

In this sense, it is well known that transversal filters have several advantages over other inline topologies. The main one is that a maximum number of transmission zeros can be implemented for a given order of the filter. Another advantage is that compact topologies can usually be implemented due to the relatively easy coupling routing scheme used. Apart from the advantages of transversal filters, this topology is known to be more sensitive to mechanical tolerances. This can be a drawback for certain high-precision applications, which, in general, can be overcome by introducing tuning elements in the final filter structure.

Synthesis methods for transversal filters were first presented in [7]. Since then, several topologies were proposed, both in printed [8] and waveguide [9] technology. However, the implementation of transversal filters using inductive waveguide structures was only proposed in [6]. In that study, the use of the modes $\mathrm{TE}_{102}$ and $\mathrm{TE}_{201}$ was proposed as the fundamental resonances of the filter. The main drawback of this structure is that the volume of the resonant cavity need to be increased (larger cavity width) in order to allow for the propagation of the higher order $\mathrm{TE}_{20}$ mode of the waveguide.

In this paper, we present an alternative method for the implementation of transversal filters using inductive waveguide configurations. The structure is formed with a cavity coupled with inductive windows, and with a dielectric post placed asymmetrically inside the cavity. The resulting topology is very compact in size since the width of the cavity is not increased to allow for the propagation of higher order modes. We show in this paper that the dimensions of both the waveguide cavity and dielectric post can control all the coupling parameters needed to synthesize useful transfer functions. This novel topology for filters allows to easily obtain high selective elliptic filters saving mass and volume. Results obtained with the transversal coupling matrix theory described in [7] are compared with simulations obtained with the Ansoft's finite-element High Frequency Structure Simulator (HFSS) tool and with an integral-equation technique, validating the new topology proposed.

\section{DEsCRIPTION OF THE Filter TOPOLOGY}

The filter under study consists of the classical doublet topology shown in Fig. 1. This structure is known to have a response with one transmission zero if no coupling between the source and the load is considered [8], [10]. The proposed practical topology for implementing this type of filter is sketched in Fig. 2. It consists of a cavity delimited by two inductive windows with a square dielectric post joined to the center of one of the cavity walls. These two elements (cavity and dielectric post) are the two basic components of the filter, which will allow the combination of the two resonances $R_{1}$ and $R_{2}$ of the doublet scheme in Fig. 1. The volume of the waveguide resonator is also reduced by the presence of the dielectric post. In addition, the width of the waveguide need not be increased to allow for the propagation of higher order modes. This represents a 


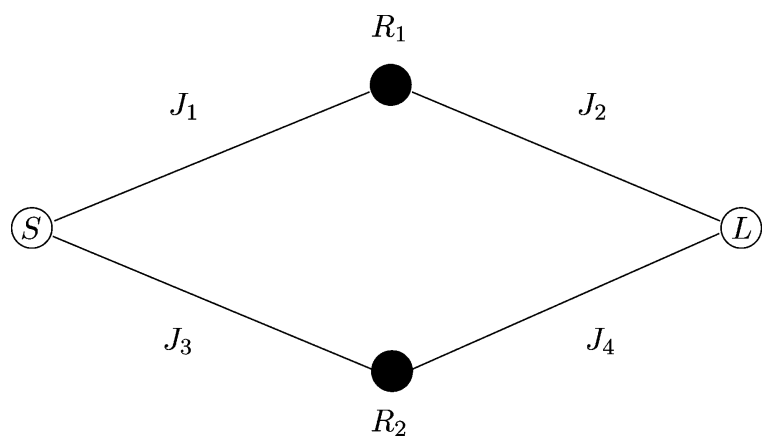

Fig. 1. Typical routing scheme of a doublet. $J_{1}$ to $J_{4}$ represents the couplings between the source $S$ and the load $L$ to the resonators $R_{1}$ and $R_{2}$.

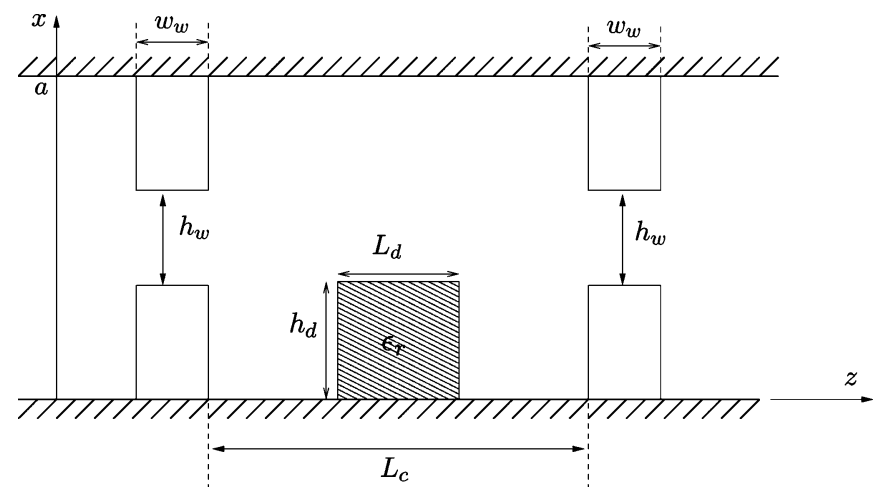

Fig. 2. Proposed topology for implementing the doublet scheme ofFig. 1. The structure consists of a single cavity with a square dielectric post attached to one of the waveguide walls.

TABLE I

Coupling MATRIX $M$ OF THE Doublet SHOWn IN Fig. 1

\begin{tabular}{|c||c|c|c|c|}
\hline & $S$ & $R_{1}$ & $R_{2}$ & $L$ \\
\hline \hline$S$ & 0 & $J_{1}$ & $J_{3}$ & 0 \\
\hline$R_{1}$ & $J_{1}$ & $M_{11}$ & 0 & $J_{2}$ \\
\hline$R_{2}$ & $J_{3}$ & 0 & $M_{22}$ & $J_{4}$ \\
\hline$L$ & 0 & $J_{2}$ & $J_{4}$ & 0 \\
\hline
\end{tabular}

further reduction of the volume of the device, as compared to the original design presented in [6].

Following the theory of the transversal filter topology presented in [7], the coupling matrix $M$ of the doublet in Fig. 1 is known to be of the form shown in Table I. In this table, $S$ is the input port (source) and $L$ is the output port (load). For our proposed topology, the elements of the coupling matrix $M$ can be controlled by means of the dimensions shown in Fig. 2.

The first resonator of the doublet is formed with the $\mathrm{TE}_{102}$ mode of the waveguide cavity formed between the two inductive steps. To demonstrate that the $\mathrm{TE}_{102}$ mode is actually resonating in the structure, in Fig. 3 we present the electric field distribution inside an empty cavity resonator of length $L_{c}=$ $19.9 \mathrm{~mm}$. We can observe that the electric field has two variations along the length of the cavity, typical of second-order resonances. In Fig. 4, we present a wide frequency sweep of the response of this cavity. We can clearly observe the first-order resonance at $9.8 \mathrm{GHz}$ and the second-order resonance at $14 \mathrm{GHz}$. It is at this frequency that we will operate the final filter. It is

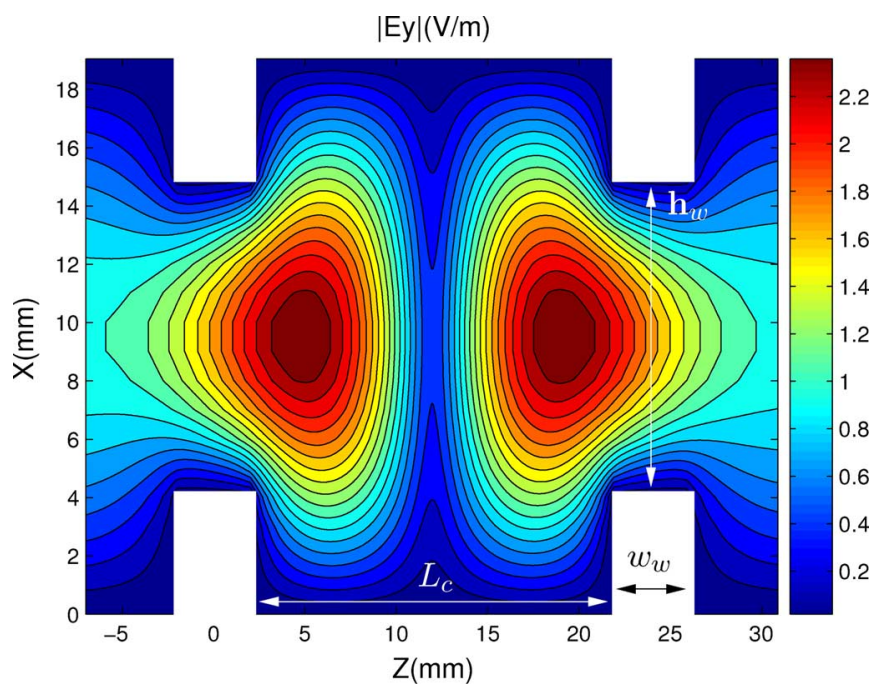

Fig. 3. Electric field distribution inside the cavity without the dielectric post, showing the typical field distribution of the $\mathrm{TE}_{102}$ mode. Dimensions: $a=$ $19.05 \mathrm{~mm}, L_{c}=19.9 \mathrm{~mm}, w_{w}=4 \mathrm{~mm}, h_{w}=10.65 \mathrm{~mm}, f=14 \mathrm{GHz}$.

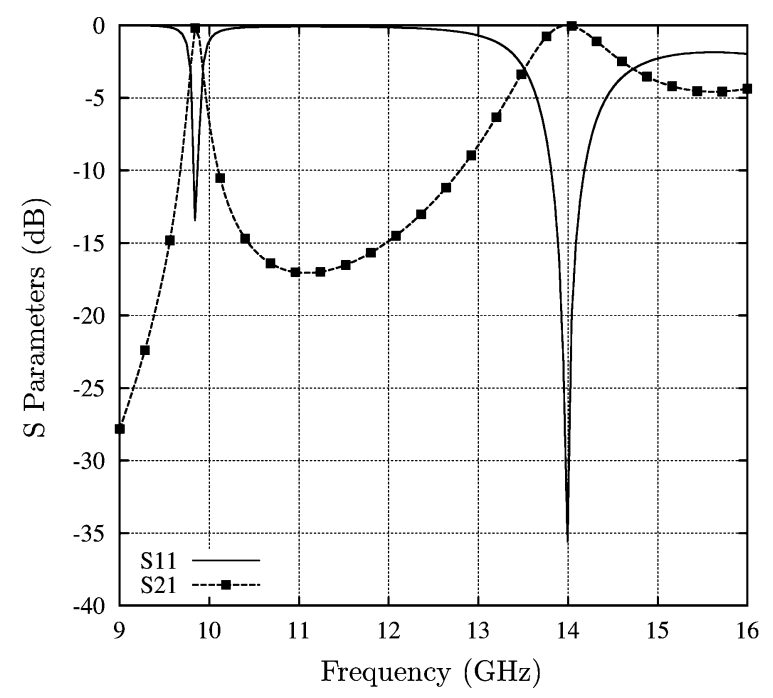

Fig. 4. Wide frequency sweep with the $S$-parameters of the cavity shown in Fig. 3.

also important to note that the use of the second-order resonance along the cavity length is essential for the correct operation of the filter. This is because in the doublet topology shown in Fig. 1, one of the four couplings must always be opposite in sign with respect to the sign of the other three couplings [11]. The change in sign of the electric field shown in Fig. 3 is actually implementing this negative coupling. Considering the $\mathrm{TE}_{102}$ mode as the first resonator $\left(R_{1}\right)$ of the filter, $J_{1}$ and $J_{2}$ are the impedance inverter constants associated to the couplings between this resonator to the source and load, respectively. From the above discussion, it is simple to see that the amount of coupling to this resonance is controlled with the width of the inductive windows ( $h_{w}$ in Fig. 2).

With the length of the cavity selected in the previous example, the $\mathrm{TE}_{103}$ mode cannot resonate in the cavity. However, since the basic mode $\mathrm{TE}_{102}$ has a zero field at the center of the cavity, we can place a dielectric post there without introducing important perturbations to this resonance. On the contrary, the field of 


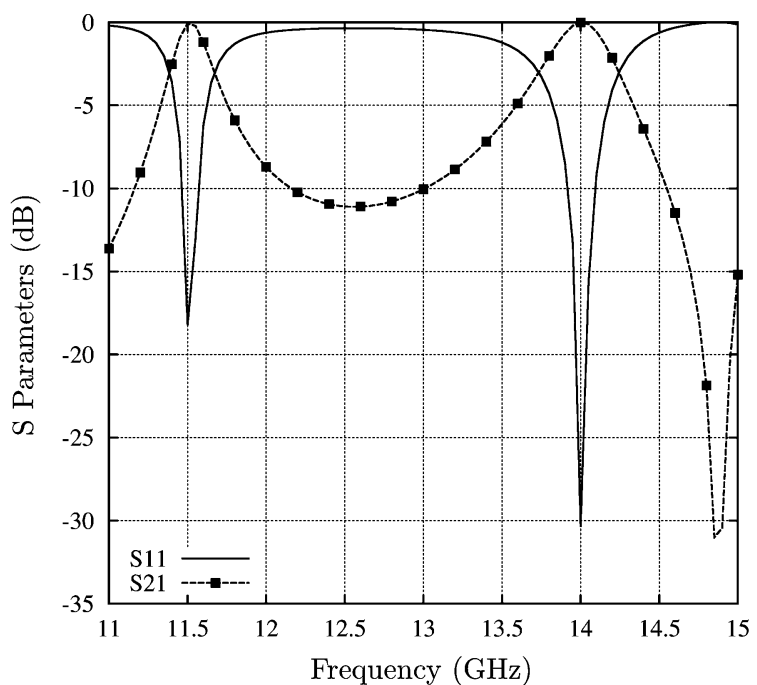

Fig. 5. Scattering parameters of a cavity loaded with a dielectric post. Dimensions according to Fig. 2: $a=19.05 \mathrm{~mm}, L_{c}=30 \mathrm{~mm}, w_{w}=4 \mathrm{~mm}$, $h_{w}=10.65 \mathrm{~mm}, L_{d}=3.5 \mathrm{~mm}, h_{d}=4 \mathrm{~mm}, \epsilon_{r}=4$.

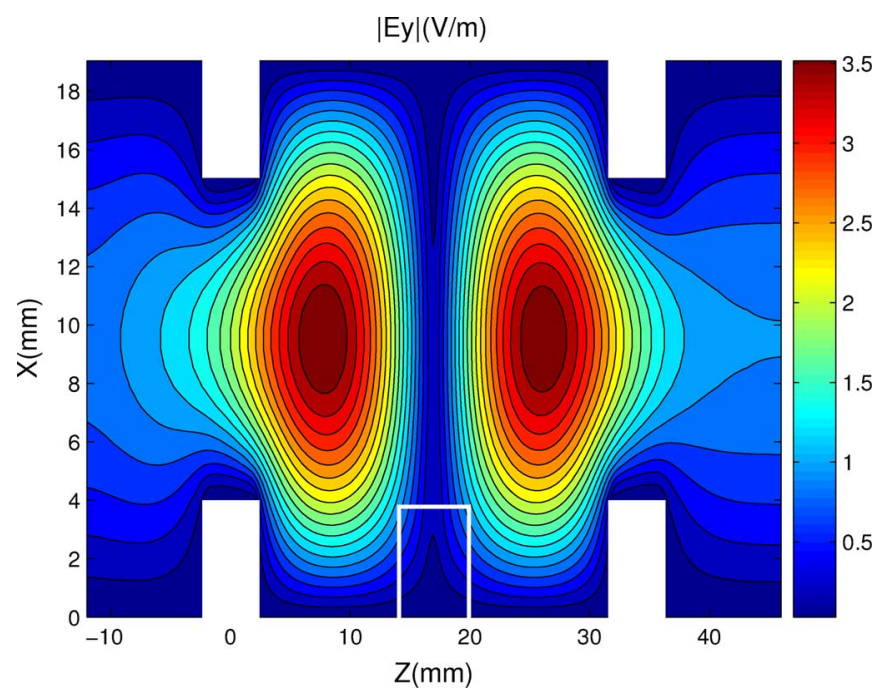

Fig. 6. Resonant $\mathrm{TE}_{102}$ mode in the cavity loaded with a dielectric post at a frequency of $11.5 \mathrm{GHz}$. Dimensions according to Fig. 2: $a=19.05 \mathrm{~mm}$, $L_{c}=30 \mathrm{~mm}, w_{w}=4 \mathrm{~mm}, h_{w}=10.65 \mathrm{~mm}, L_{d}=3.5 \mathrm{~mm}, h_{d}=4 \mathrm{~mm}$, $\epsilon_{r}=4$.

the $\mathrm{TE}_{103}$ mode is maximum at the center of the cavity. Therefore, a dielectric post can be used to strongly lower its resonant frequency to the required design frequency of the filter.

To illustrate these concepts, we have analyzed a similar cavity as before, but including a dielectric post of relative permittivity $\epsilon_{r}=4$. The length of the cavity is also increased to $L_{c}=$ $30 \mathrm{~mm}$ to de-tune the $\mathrm{TE}_{102}$ resonance of the cavity. In Fig. 5, we present the scattering parameters obtained for this structure in a wide frequency sweep. We can observe a first resonance of the cavity at $11.5 \mathrm{GHz}$. This is the $\mathrm{TE}_{102}$ resonance, which is now at lower frequencies due to the larger cavity size used. This is demonstrated by looking at the field pattern obtained at 11.5 GHz, presented in Fig. 6. We again observe the field pattern of the $\mathrm{TE}_{102}$ mode without practically any disturbance caused by the dielectric post.

The important point to discuss now is that a second resonance appears in Fig. 5 at $14 \mathrm{GHz}$. This resonance is due to the $\mathrm{TE}_{103}$

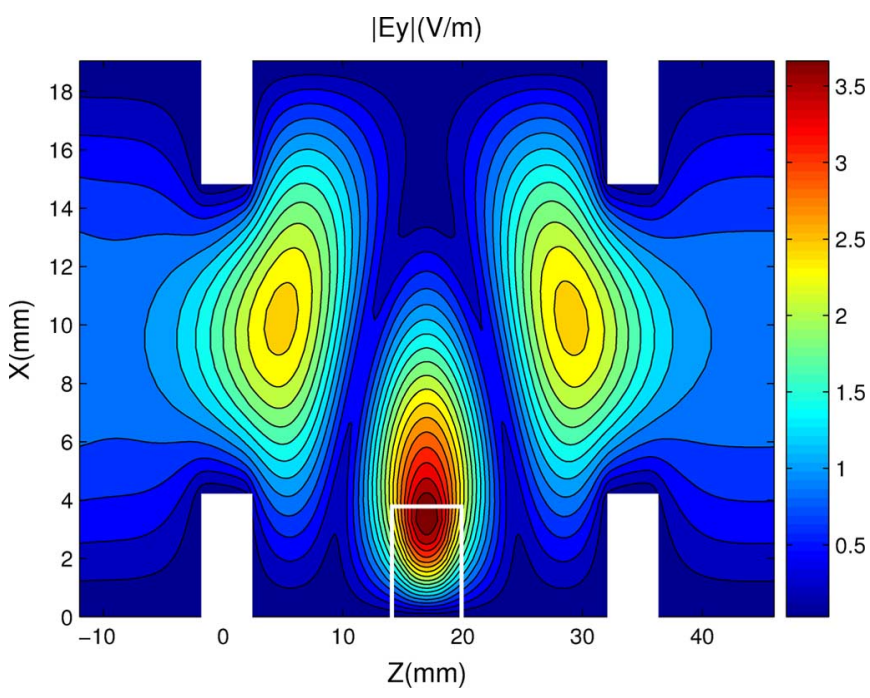

Fig. 7. Resonant $\mathrm{TE}_{103}$ mode perturbed by the dielectric post at the frequency of $14 \mathrm{GHz}$. Dimensions according to Fig. 2: $a=19.05 \mathrm{~mm}, L_{c}=30 \mathrm{~mm}$, $w_{w}=4 \mathrm{~mm}, h_{w}=10.65 \mathrm{~mm}, L_{d}=3.5 \mathrm{~mm}, h_{d}=4 \mathrm{~mm}, \epsilon_{r}=4$.

mode, which has a lower resonance frequency due to a strong interaction with the dielectric post placed in the middle of the cavity. To demonstrate that this is indeed a perturbed $\mathrm{TE}_{103}$ resonant mode, we further present in Fig. 7 the electric field pattern of the structure at $14 \mathrm{GHz}$. We can observe the three typical lobes of a third-order resonance. We also observe that the central lobe is concentrated inside the dielectric, therefore strongly affecting the resonant frequency of the mode. In the final filter, the $\mathrm{TE}_{103}$ resonance will act as the second resonance of the filter ( $R_{2}$ of Fig. 1 ), and it will be combined with the $\mathrm{TE}_{102}$ mode resonance $\left(R_{1}\right.$ of Fig. 1$)$ to build the final doublet. Finally, note that the electric field of the $\mathrm{TE}_{103}$ mode suffers two sign changes along the cavity length, as shown in Fig. 7. The signs of the couplings associated to this resonance then do not change from input to output. This is in accordance with the fact that only one sign of the four involved in the doublet must change in sign. It is also important to bear in mind that the presence of this dielectric post will hardly disturb the first $\mathrm{TE}_{102}$-mode resonance of the final filter, as demonstrated in Fig. 6. The disturbance comes in a slight modification of the resonant frequency of the mode. This effect can be easily compensated by adjusting back (reducing) the length of the waveguide cavity $L_{c}$ (see Fig. 2).

Following this concept, it is simple to see that the inductive windows of the cavity will also affect the coupling to the second mode of the doublet. However, once this coupling window is fixed, the coupling to the second mode of the doublet can be further adjusted with the height of the dielectric post. This change will not modify the behavior of the $\mathrm{TE}_{102}$ mode. However, the change of this height will modify the pulling effect of the central lobe of the $\mathrm{TE}_{103}$, therefore effectively modifying the coupling. The corresponding impedance inverters associated to these couplings are $J_{3}$ and $J_{4}$ according to the coupling matrix of Table I (see also Fig. 1).

Finally, the length of the cavity $L_{c}$ will modify the resonant frequencies of both modes. However, once the resonant frequency of the $\mathrm{TE}_{102}$ mode is adjusted with this length, the resonant frequency of the $\mathrm{TE}_{103}$ mode can be further adjusted by modifying the length $L_{d}$ of the dielectric post. The differences in the resonant frequencies of both resonators in asynchronously 


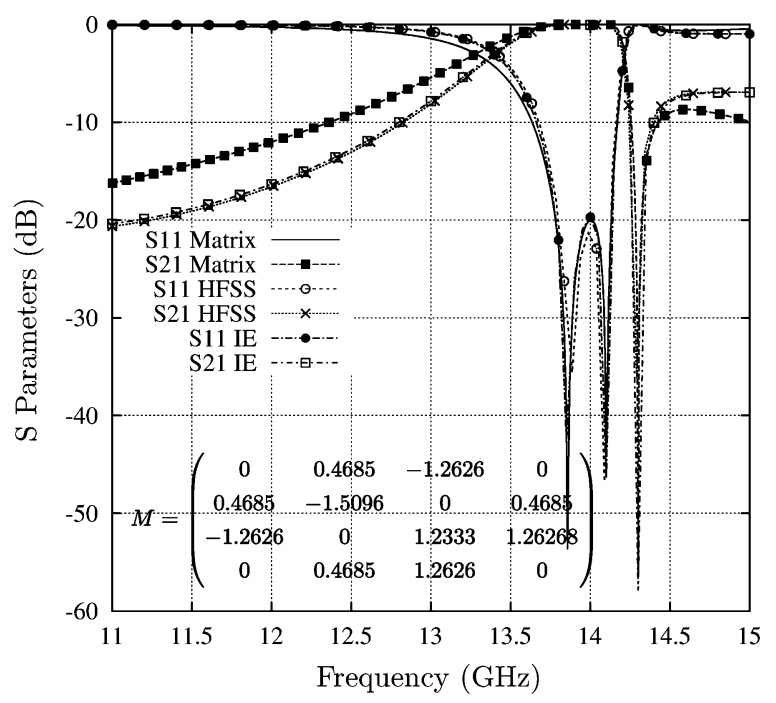

Fig. 8. Coupling matrix and scattering parameters of a bandpass filter with a transmission zero above the passband. The waveguide width is $a=19.05 \mathrm{~mm}$. The dimensions according to Fig. 2 are: $w_{w}=4 \mathrm{~mm}, h_{w}=10.65 \mathrm{~mm}$, $h_{d}=4.1 \mathrm{~mm}, L_{d}=5 \mathrm{~mm}, L_{c}=20.4 \mathrm{~mm}, \epsilon_{r}=4$.

tuned filters are included in the self-coupling terms $M_{11}$ and $M_{22}$ of the coupling matrix shown in Table I [7].

With these simple considerations, it is easy to optimize the dimensions of the filter in order to synthesize the values of any coupling matrix of the form shown in Table I. As already stated, we observe that, in this case, there is no need to increase the width of the waveguide cavity $a$ in order to implement transmission zeros. This leads to more compact structures as compared to the original work proposed in [6].

\section{RESULTS}

In order to demonstrate the practical value of the proposed topology, we will consider the design of a bandpass filter centered at the frequency of $14 \mathrm{GHz}$, and with a transmission zero above the passband, at the frequency of $14.4 \mathrm{GHz}$. The filter has been optimized to exhibit a ripple within the passband of $-20 \mathrm{~dB}$. Following the synthesis technique described in [7], the coupling matrix corresponding to the structure shown in Table I takes the values presented in the inset of Fig. 8. The different geometrical parameters of the structure presented in Fig. 2 can be adjusted in order to synthesize the different values of this coupling matrix. Choosing a standard WR75 waveguide $(a=$ $19.05 \mathrm{~mm}$ ), the final dimensions for the inductive windows are $w_{w}=4 \mathrm{~mm}$ and $h_{w}=10.65 \mathrm{~mm}$. The height and length of the dielectric post are $h_{d}=4.1 \mathrm{~mm}$ and $L_{d}=5 \mathrm{~mm}$ with a relative permittivity constant $\epsilon_{r}=4$. Finally, the optimized length of the cavity is $L_{c}=20.4 \mathrm{~mm}$. The scattering parameters obtained for this geometry, when analyzed with Ansoft's commercial finite-element software HFSS, are presented in Fig. 8, together with the results obtained directly from the analysis of the coupling matrix. We can observe very good agreement between the results predicted by the coupling matrix theory and the results obtained from the full-wave analysis of the optimized structure. As a further validation test, we also show in Fig. 8 the results obtained with an integral-equation technique derived for inductive waveguide devices based on the theory presented in [12]. Again we can observe very good agreement.

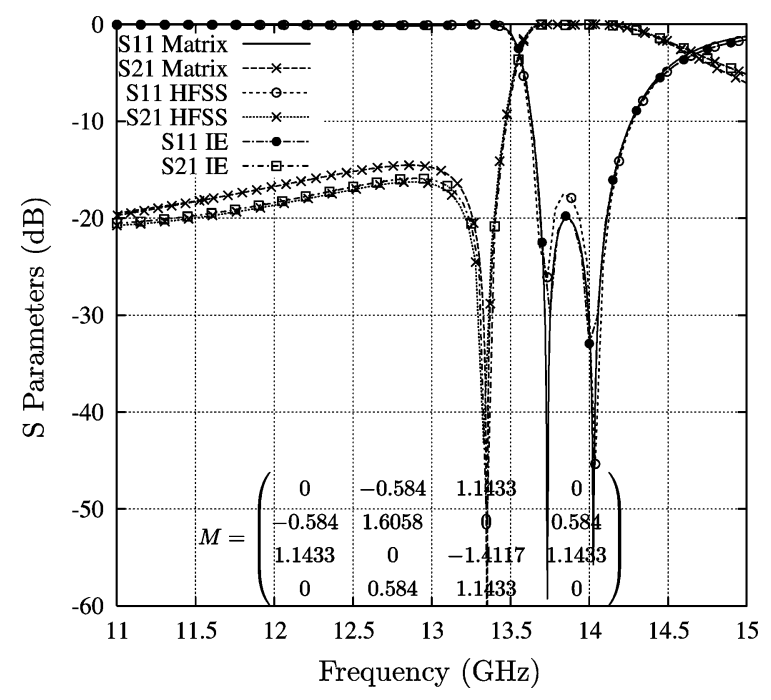

Fig. 9. Coupling matrix and scattering parameters of a bandpass filter with a transmission zero placed below the passband. The waveguide width is $a=$ $19.05 \mathrm{~mm}$. The dimensions according to Fig. 2 are: $w_{w}=4 \mathrm{~mm}, h_{w}=$ $10.95 \mathrm{~mm}, h_{d}=4.5 \mathrm{~mm}, L_{d}=8.4 \mathrm{~mm}, L_{c}=17.26 \mathrm{~mm}, \epsilon_{r}=4$.

Another interesting feature of the new topology is that it exhibits the so-called zero shifting property of transversal filters [13]. Using this property, few adjustments in the resonant frequencies of the resonators can be introduced to implement a transmission zero below the passband of the filter. To show that this is indeed the case, we present in Fig. 9 a similar filter as before, but with the transmission zero placed at the frequency of 13.3 GHz. To obtain this filtering function, only small changes were applied to the resonant frequencies of the resonators $\left(L_{c}\right.$ and $L_{d}$ ). The height $h_{d}$ was also slightly modified to compensate for the couplings to the second resonator. Finally, the input/ output windows $\left(h_{w}\right)$ were slightly adjusted to recover the return-loss level of $-20 \mathrm{~dB}$. The analysis of this new structure with Ansoft's HFSS is presented in Fig. 9, showing that the transmission zero now occurs below the passband. The inset of this figure shows the new coupling matrix synthesized with the technique presented in [7]. Results predicted by the direct analysis of this coupling matrix are also presented in Fig. 9, showing excellent agreement with the full-wave simulations of the structure. Again, results obtained with the integral-equation technique based on the theory described in [12] are also shown for further validation.

It is worth mentioning that the doublets designed in this paper can be cascaded together to produce higher order filtering functions, as originally proposed in [14]. This can be done by using a nonresonating node as the basic element to join the doublets [15]. Alternatively, doublets can be cascaded by using conjoining resonators (rather than nonresonating nodes). To do this, trisections are first created, and then cross-pivot rotations are applied to form the cascaded doublets (see [11, Fig.7]).

From the results shown in Figs. 8 and 9, we observe that, in the new structure proposed, the transmission zeros are placed close to the passband. This is due to the fact that the coupling from the ports to the $\mathrm{TE}_{102}$ mode is stronger than the coupling to the $\mathrm{TE}_{103}$ mode. This can also be verified from the field patterns shown in Figs. 6 and 7. The $\mathrm{TE}_{102}$ mode has most of its strength close to the ports, while the modified $\mathrm{TE}_{103}$ mode is concentrated in the dielectric post at the center of the cavity. 


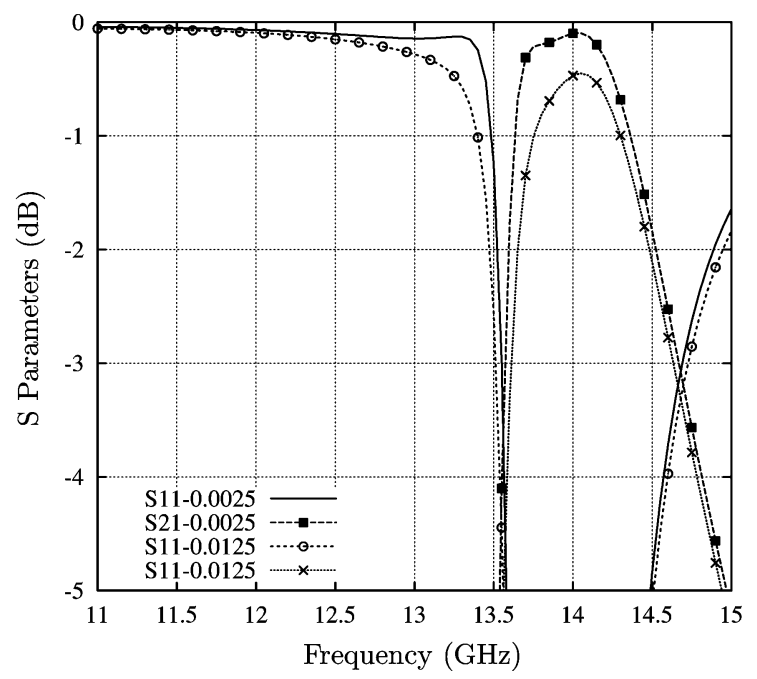

Fig. 10. Details of the insertion losses of the filter of Fig. 9 for two different values of the dielectric loss tangent: $\tan \delta=0.0025$ and $\tan \delta=0.0125$.

Consequently, the coupling from the ports to the $\mathrm{TE}_{102}$ mode will always be larger, resulting in transmission zeros close to the passband. However, the strength of the coupling from the input/output ports to the resonators is essentially controlled by the coupling windows ( $h_{w}$ in Fig. 2). This means that large couplings can be synthesized for the design of wide bandpass filters. In the examples shown in Figs. 8 and 9, the filters exhibit bandwidths of $400 \mathrm{MHz}$ with return losses of $-20 \mathrm{~dB}$. Of course, narrower bandpass filters can also be designed by closing the input/output windows to reduce the strength of the couplings.

Another interesting aspect of the topology proposed is related to the insertion losses that can be achieved with this structure. From the behavior of the resonances of the filter presented in Figs. 6 and 7, we can observe that the quality factor of the $\mathrm{TE}_{102}$ mode will essentially be the same as in a conventional $H$-plane filter. This is because this mode is hardly affected by the dielectric post. On the contrary, the quality factor of the $\mathrm{TE}_{103}$ mode will strongly depend on the losses of the dielectric object since this mode has most of its strength inside the post, as shown in Fig. 7. To demonstrate this fact, we include in Fig. 10 the details of the insertion-loss response of the filter presented in Fig. 9, when the losses in the dielectric post are increased. This figure shows a slope in the insertion-loss response of the filter, being maximum around the frequency of $13.7 \mathrm{GHz}$, where the $\mathrm{TE}_{103}$ mode has its strongest effect. For a value $\tan \delta=0.0125$, the insertion loss of the filter varies inside the passband from $-1.3 \mathrm{~dB}$ (at the worst point) to $-0.4 \mathrm{~dB}$. When $\tan \delta=0.0025$, the insertion loss improves from $-0.3 \mathrm{~dB}$ (at the worst point) to only $-0.1 \mathrm{~dB}$.

Finally, it is important to notice that the out-of-band response of the transversal filter proposed in this paper is expected to be worse than in a conventional $H$-plane filter. This is because of the influence of the dielectric post in the higher order modes of the resonator. Thanks to the dielectric post, we can combine the $\mathrm{TE}_{102}$ and $\mathrm{TE}_{103}$ modes to make a transversal filter. The $\mathrm{TE}_{103}$ mode is pulled down to lower frequencies due to the presence of the dielectric post. However, any other higher order mode having a maximum at the center of the cavity (for instance, the $\mathrm{TE}_{105}$ mode), will also be pulled down to lower frequencies by the dielectric post. This will certainly approach the spurious

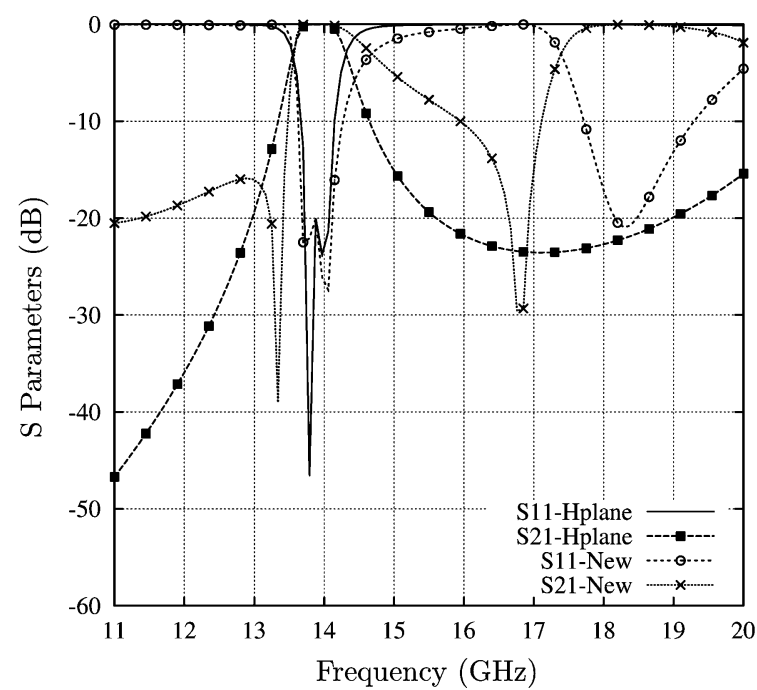

Fig. 11. Comparison of the out-of-band performance for the new transversal filter against a standard $H$-plane filter of similar characteristics.

bands closer to the useful operational band. To demonstrate this, we have designed a similar two pole filter in a standard $H$-plane technology, and we compare them with the filter shown in Fig. 9. The results of the comparison are presented in Fig. 11. We observe that the first spurious band is obtained at the frequency of $18 \mathrm{GHz}$ in the new filter. However, the conventional $H$-plane filter has the first spurious band at $22 \mathrm{GHz}$.

\section{CONCLUSIONS}

In this paper, we have proposed a new topology for implementing high-selectivity bandpass filters with one transmission zero in its insertion-loss response. The structure is formed with a waveguide cavity and a dielectric resonator attached to one of the waveguide walls. This paper has shown that this structure is able to implement a transversal filter topology (doublet) with reduced size and volume. This topology produces a single transmission zero, which can be easily placed above or below the passband using the zero shifting principle. Results obtained through full-wave analysis of the new structure proposed are in good agreement with theoretical results, which have been directly obtained with the coupling matrix theory.

\section{REFERENCES}

[1] M. Mokhtaari, J. Bornemann, K. Rambabu, and S. Amari, "Coupling matrix design of dual and triple passband filters," IEEE Trans. Microw. Theory Tech., vol. 54, no. 11, pp. 3940-3946, Nov. 2006.

[2] M. Guglielmi, G. Gheri, and A. A. Melcon, "CAD of tuning-less bandpass filters," ESA-ESTEC, Noordwijk, The Netherlands, ESTEC Working Paper 1624, Jul. 1991.

[3] V. E. Boria, M. Bozzi, D. Camilleri, A. Coves, H. Esteban, B. Gimeno, M. Guglielmi, and L. Polini, "Analysis and design of all-inductive filters with dielectric resonators," in Proc. 33rd Microw. Conf., Munich, Germany, Oct. 7-9, 2003, vol. 3, pp. 1247-1250.

[4] Y. Leviatan, P. G. Li, A. T. Adams, and J. Perini, "Single post inductive obstacle in rectangular waveguide," IEEE Trans. Microw. Theory Tech., vol. MTT-31, no. 10, pp. 806-812, Oct. 1983.

[5] P. G. Li, A. T. Adams, Y. Leviatan, and J. Perini, "Multiple post inductive obstacles in rectangular waveguide," IEEE Trans. Microw. Theory Tech., vol. MTT-32, no. 4, pp. 365-373, Apr. 1984.

[6] M. Guglielmi, P. Jarry, E. Kerherve, O. Roquebrun, and D. Schmitt, "A new family of all-inductive dual-mode filters," IEEE Trans. Microw. Theory Tech., vol. 49, no. 10, pp. 1764-1769, Oct. 2001.

[7] R. J. Cameron, "Advanced coupling matrix synthesis techniques for microwave filters," IEEE Trans. Microw. Theory Tech., vol. 51, no. 1, pp. 1-10, Jan. 2003. 
[8] D. C. Rebenaque, A. A. Melcon, and M. Guglielmi, "A new simple microstrip open-loop resonators filter for high selectivity applications," in IEEE MTT-S Int. Microw. Symp. Dig., Philadelphia, PA, Jun. 8-13, 2003, pp. 1603-1606, Paper TH-1B-3.

[9] S. Amari, U. Rosenberg, and J. Bornemann, "Adaptive synthesis and design of resonator filters with source/local-multiresonator couplings," IEEE Trans. Microw. Theory Tech., vol. 50, no. 8, pp. 1969-1975, Aug. 2002.

[10] S. Amari and U. Rosenberg, "A universal building block for advanced modular design of microwave filters," IEEE Microw. Wireless Compon. Lett., vol. 13, no. 12, pp. 541-543, Dec. 2003.

[11] R. J. Cameron, A. R. Harish, and C. J. Radcliffe, "Synthesis of advanced microwave filters without diagonal cross-coupling," IEEE Trans. Microw. Theory Tech., vol. 50, no. 12, pp. 2862-2872, Dec. 2002.

[12] F. Quesada-Pereira, F. Pérez-Soler, B. Gimeno-Martínez, V. E. Boria-Esbert, J. Pascual-García, J. L. Gómez-Tornero, and A. Álvarez-Melcón, "Efficient analysis tool of inductive passive waveguide components and circuits using a novel space domain integral formulation," in Proc. Eur. Microw. Conf., Manchester, U.K., Sep. 11-15, 2006, pp. 1003-1006.

[13] D. C. Rebenaque, F. Q. Pereira, J. P. Garcia, A. A. Melcon, and M. Guglielmi, "Two compact configurations for implementing transmission zeros in microstrip filters," IEEE Microw. Wireless Compon. Lett., vol. 14, no. 10, pp. 475-477, Oct. 2004.

[14] U. Rosenberg and S. Amari, "Novel coupling schemes for microwave resonator filters," IEEE Trans. Microw. Theory Tech., vol. 50, no. 12, pp. 2896-2902, Dec. 2003.

[15] S. Amari and U. Rosenberg, "New building blocks for modular design of elliptic and self-equalized filters," IEEE Trans. Microw. Theory Tech., vol. 52, no. 2, pp. 721-736, Feb. 2004.

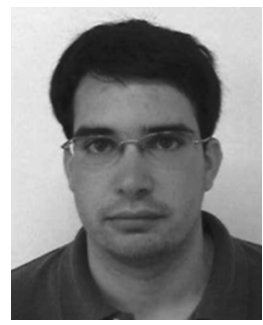

Francisco Javier Pérez Soler (S'05) was born in Murcia, Spain, in 1981. He received the Telecommunications Engineer degree from the Technical University of Cartagena (UPCT), Cartagena, Spain, in 2004, and is currently working towards the Ph.D. degree at UPCT.

He is currently with the Communication and Information Technologies Department, UPCT. His current scientific interests include the integral-equation technique for the analysis of antennas and microwave devices.

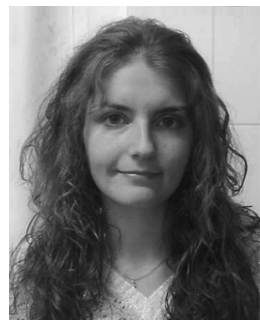

Mónica Martínez Mendoza was born in Cartagena Murcia, Spain, in 1983. She received the Telecommunications Engineer degree from the Technical University of Cartagena (UPCT), Cartagena, Spain, in 2006, and is currently working toward the Ph.D. degree at UPCT.

In 2007, she joined the Telecommunications and Electromagnetic Group, UPCT, as a Research Assistant, where she is involved in the development of novel transversal filtering structures for satellite analysis and design of microwave circuits. systems. Her current scientific interests include the

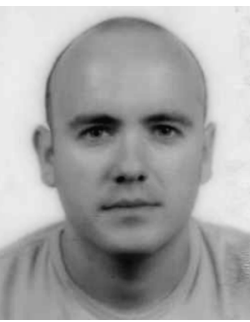

Fernando Daniel Quesada Pereira (S'05-M'07) was born in Murcia, Spain, in 1974. He received the Telecommunications Engineer degree from the Technical University of Valencia (UPV), Valencia, Spain, in 2000, and the Ph.D. degree from the Technical University of Cartagena, Cartagena, Spain in 2007.

In 1999, he joined the Radiocommunications Department, UPV, as a Research Assistant, where he was involved in the development of numerical methods for the analysis of anechoic chambers and tag antennas. In 2001, he joined the Technical University of Cartagena, Cartagena, Spain, initially as an Research Assistant and then becoming an
Assistant Professor. His current scientific interests include the integral-equation technique applied to the analysis of antennas and microwave devices.

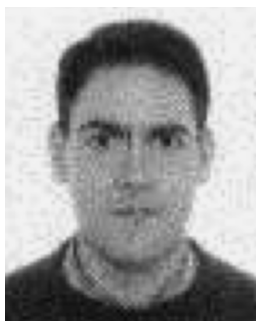

David Cañete Rebenaque (S'04) was born in Valencia, Spain, in 1976. He received the Telecommunications Engineer degree from the Technical University of Valencia, Valencia, Spain, in 2000, and is currently working toward the Ph.D. degree at the University of Cartagena, Cartagena, Spain.

During 2001, he was an RF Engineer with a mobile communication company. In 2002 , he joined the Communications and Information Technologies Department, Technical University of Cartagena. His research interests include analysis and design of microwave circuits and active antennas.

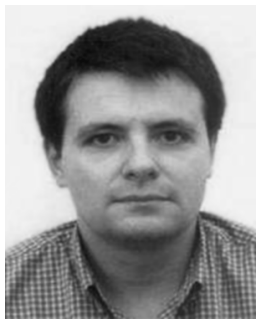

Alejandro Alvarez Melcon (M'99-SM'07) was born in Madrid, Spain, in 1965. He received the Telecommunications Engineer degree from the Technical University of Madrid (UPM), Madrid, Spain, in 1991, and the Ph.D. degree in electrical engineering from the Swiss Federal Institute of Technology, Lausanne, Switzerland, in 1998.

In 1988, he joined the Signal, Systems and Radiocommunications Department, UPM, as a Research Student, where he was involved in the design, testing, and measurement of broadband spiral antennas for electromagnetic measurements support (EMS) equipment. From 1991 to 1993, he was with the Radio Frequency Systems Division, European Space Agency (ESA)/European Space Research and Technology Centre (ESTEC), Noordwijk, The Netherlands, where he was involved in the development of analytical and numerical tools for the study of waveguide discontinuities, planar transmission lines, and microwave filters. From 1993 to 1995, he was with the Space Division, Industry Alcatel Espacio, Madrid, Spain, and also with the ESA, where he collaborated on several ESA/ESTEC contracts. From 1995 to 1999, he was with the Swiss Federal Institute of Technology, École Polytechnique Fédérale de Lausanne (EPFL), Lausanne, Switzerland, where he was involved in the field of microstrip antennas and printed circuits for space applications. In 2000, he joined the Technical University of Cartagena, Cartagena, Spain, where he currently develops his teaching and research activities.

Dr. Alvarez Melcón was the recipient of the Journée Internationales de Nice Sur les Antennes (JINA) Best Paper Award for the best contribution to the JINA'98 International Symposium on Antennas, and the Colegio Oficial de Ingenieros de Telecomunicación (COIT/AEIT) Award for the best doctoral thesis in basic information and communication technologies.

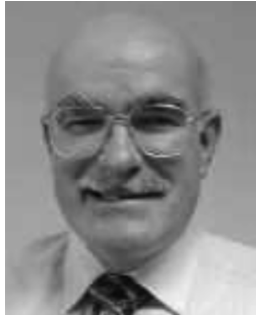

Richard J. Cameron (M'83-SM'94-F'02) was born in Glasgow, U.K., in 1947. He received the B.Sc. degree in telecommunications and electronic engineering from Loughborough University, Loughborough, U.K., in 1969.

In 1969, he joined Marconi Space and Defence Systems, Stanmore, U.K., where his research activities included small earth-station design, telecommunication satellite system analysis, and computer-aided RF circuit and component design. In 1975, he joined the European Space Agency (ESA)/European Space Research and Technology Centre (ESTEC), Noordwijk The Netherlands, where he was involved in the research and development of advanced microwave active and passive components and circuits with applications in telecommunications, scientific, and earth observation spacecraft. In 1984, he joined COM DEV International Ltd., Aylesbury, U.K., where he has been involved in the software and methods for the design of a wide range of high-performance components and subsystems for both space and terrestrial application.

Mr. Cameron is a Fellow of the Institution of Electrical Engineers (IEE), U.K. 